\title{
EFFETS DE CERTAINS AGENTS ANESTHESIQUES SUR L'ACTIVITE ELECTRIQUE CEREBRALE
}

\author{
G. Vourc'h, M.D., G. Arfel, M.D., et J. M. Besson, M.D."
}

BIEN que L'ANESTHÉSIE gÉNÉRALE soit pratiquée tous les jours, dans tous les hôpitaux, à l'aide d'agents fort divers (gaz, vapeurs, injections intraveineuses), la nature même de l'anesthésie reste mystérieuse. Le symposium de $1950^{1}$ a fait le point sur l'état de nos connaissances dans ce domaine. Depuis, une nouvelle théorie, celle de Pauling, a tenté d'expliquer l'action des drogues anesthésiques ${ }^{2}$ par un schéma différent. Ces théories, pour intéressantes qu'elles soient, restent fragmentaires et ne rendent pas compte de l'ensemble des phénomènes observés. L'anesthésie affecte certainement toutes les cellules de l'organisme; on a pu l'appliquer à des êtres monocellulaires, à des plantes, dépourvues de système nerveux. Toutefois, on admet que sur les animaux et sur l'homme, son action s'exerce électivement sur le système nerveux central. La loi de Hughlings-Jackson, en général vérifiée en clinique, précise que les structures nerveuses les plus récentes philogénétiquement sont les plus sensibles à l'action des anesthésiques. ${ }^{3}$

De nombreux travaux ont fait état de l'action de l'anesthésie générale, induite par divers agents, sur l'électroencéphalogramme.4-6 Mais l'électroencéphalogramme ne recueille que l'activité corticale transmise au travers de la boite cranienne et du scalp, et donc très atténuée.

En neurochirurgie, et en particulier en neurochirurgie stéréotaxique, le problème de l'enregistrement des activités corticales et thalamiques est fondamental. On peut avoir à réséquer une zone épileptogène, en respectant le cortex sain; ou à exciser une région limitée dans le traitement d'algies rebelles (topectomie). Certaines dyskynésies, et en particulier les maladies de Parkinson, posent des problèmes délicats: la localisation de la région à détruire, dans le thalamus, dépend de son repérage à partir de données anatomiques; mais comme Guiot ${ }^{7}$ l'a montré, ces éléments sont imprécis et variables d'un sujet à l'autre; par contre la localisation peut en être faite avec une grande précision en utilisant des techniques neurophysiologiques: une électrode bipolaire enregistre les activités, spontanées ou époguées, des diverses structures rencontrées, et permet un repérage exact. ${ }^{8-11}$

Ces interventions sont souvent longues et inconfortables pour le malade; en outre, certains mouvements anormaux (tremblements) peuvent gêner l'enregistrement. Il est donc apparu qu'il était souhaitable, et parfois nécessaire, de recourir soit à une anesthésie générale, soit à une sédation satisfaisante.

Or, les drogues anesthésiques modifient considérablement l'électrogénèse cérébrale, au point de rendre parfois l'interprétation du tracé impossible. Il a donc

'Dr. Vourc'h est Professeur d'anesthésiologie, Faculté de médecine, Paris, et Chef du service d'anesthésie, Hôpital Foch, Suresnes Seine. Dr. Arfel est Maître de recherches, I.N.S.E.R.M., et Chef du laboratoire d'électroencéphalographie, Hôpital Foch, Suresnes Seine. Dr. Besson est Attaché de recherches, C.N.R.S., et avec le Centre d'études de physiologie nerveuse, Paris. 
fallu étudier expérimentalement, sur le chat et le singe, puis cliniquement sur l'homme, l'action de la plupart de ces drogues, afin d'opérer une sélection et de ne retenir que celles qui sont compatibles avec des conditions opératoires satisfaisantes.

\section{ETUDE EXPÉRIMENTALE}

Tant en expérimentation qu'en clinique, il est difficile d'étudier les effets d'une drogue isolée; on a le plus souvent recours à des associations (anesthésiques volatils au intraveineux; analgésiques; myorésolutifs). S'il est indispensable d'étudier les actions de chaque agent pour en recueillir les effets dans toute leur pureté, il convient également de considérer les interactions possibles avec d'autres agents, lorsqu'ils sont combinés.

Denavit ${ }^{12}$ a effectué une étude très complète de certains anesthésiques utilisés en clinique. Elle a pu classer ces drogues en deux groupes, selon leurs actions sur les centres thalamiques et corticaux primaires ou associatifs:

-Quel que soit l'anesthésique, les réponses lemniscales sont en général conservées; il faut, par exemple, des concentrations d'éther importantes pour déprimer les réponses dans le noyau ventral postérieur. L'halothane (Fluothane ${ }^{\circledR}$ ) constitue une exception, en ce sens qu'il accroit l'amplitude des potentiels évoqués. Les barbituriques, le chloralose, l'hydroxydione (Viadril@), parmi les anesthésiques intraveineux étudiés, maintiennent également les réponses.

- Par contre, les réponses associatives sont bien plus sensibles à l'action des anesthésiques. Presque tous les anesthésiques les réduisent considérablement, ou même les suppriment totalement. Deux exceptions sont à relever: le chloralose et l'hydroxydione, qui les préservent.

Le cortex est en général plus rapidement déprimé que les structures profondes, ce qui est en accord avec la loi de Hughlings-Jackson.

Il est difficile de comprendre la raison de ces différences d'action; une hypothèse (Albe-Fessard, communication personnelle) serait que tous les anesthésiques affectent de la même manière les cellules nerveuses et leur synapses. Les structures primaires sont résistantes, parce qu'elles reçoivent un grand nombre d'influx: un axone aboutit à un groupe limité de cellules. Au contraire, dans les structures associatives, chaque cellule n'a que peu de boutons correspondant à une fibre afférente donnée, la dispersion est grande. A un degré d'inprégnation anesthésique égal, les influx groupés vers une cellule du système primaire franchiront le barrage; les influx dispersés destinés à une cellule du système associatif seront arrêtés. Dans le premier cas, la marge de sécurité est relativement étendue; dans le second, elle est étroite.

Ces études préliminaires ont été poursuivies et étendues, dans le but d'explorer, d'une part l'action des perturbations entraînées par divers phénomènes, respiratoires (anoxie, hyper ou hypocapnie), circulatoires (hypotension, hémorragie) sur les réponses observées; d'autre part, l'action de drogues nouvelles, non encore explorées (morphine et morphinomimétiques, anesthésiques à brève durée d'action). 


\section{ConsÉquences des PERTURBations PHySIOLOgiques}

En clinique humaine, il est bien connu que des modifications respiratoires, circulatoires, métaboliques, affectent de façon rapide et notable l'électroencéphalogramme. Aucune étude n'est possible si l'homéostasie n'est pas respectée; on observe des comas par anoxie, hyper ou hypocapnie, acidose ou alcalose, hypotension, hémorragie. ${ }^{13}$

Il nous a paru interessant de déterminer l'incidence de ces facteurs en expérimentation animale. L'opérateur doit s'assurer que la préparation sur laquelle il travaille est stable, et que les règles de la physiologie sont respectées. Toute perturbation peut modifier l'électrogénèse cérébrale, et conduire à des conclusions érronées en attribuant à une drogue ce qui n'est, en fait, que le reflet de ces perturbations.

Les expériences qui vont suivre ont été faites sur des chats, anesthésiés à l’aide de divers agents, trachéotomisés, curarisés par la gallamine, et ventilés. Un régime stable ayant été établi, diverses modifications, respiratoires ou circulatoires, ont été provoquées, et les résultats sur l'électrogénèse cérébrale enregistrés.

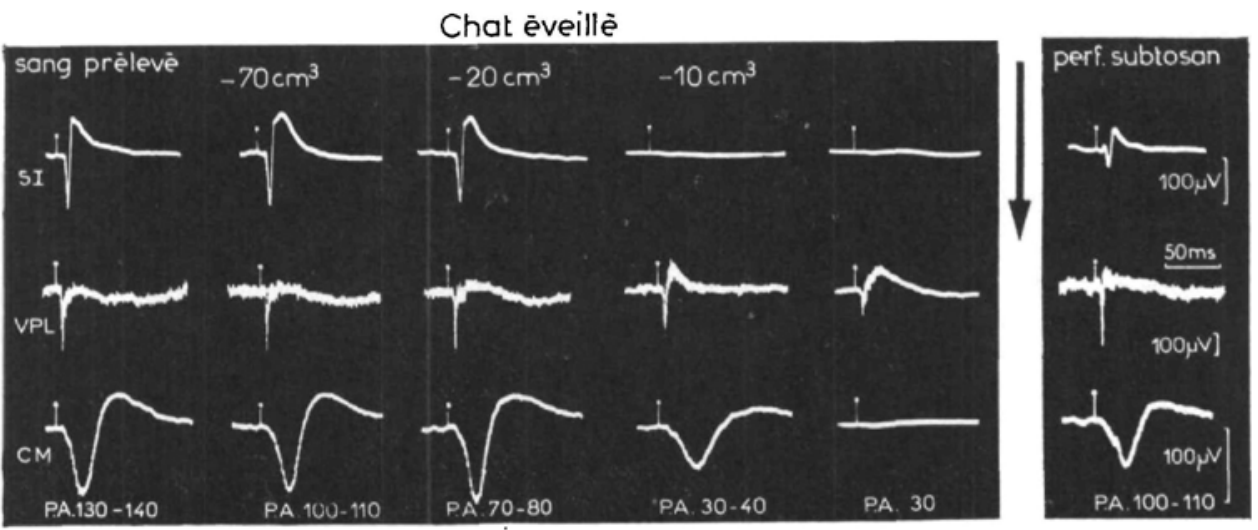

Figure 1. Chat éveillé: Modification des activités évoquées corticales et sous-corticales lors d'une spoliation sanguine. De haut en bas: cortex primaire somatique (SI), Noyau Ventro Postero Lateral du thalamus (VPL), Centre Median (CM). Jusqu'à des chiffres de pression arterielle (TA) de 70 à $80 \mathrm{~mm}$. $\mathrm{Hg}$, peu de modifications. Les réponses sont ensuite affectées dans un ordre donné, toujours le même: cortex, CM, VPL. Une perfusion de solution macromoléculaire (Subtosan( ${ }^{(1)}$ ramène la $\mathrm{TA}$ à un niveau normal et permet une récupération dans le VPL; mais les réponses dans le SI et le CM restent déprimées.

\section{Anoxie}

L'anoxie peut revêtir diverses formes (anoxique, anémique, circulatoire, histotoxique); dans tous les cas, elle a pour résultat un défaut d'apport d'oxygène, ou de sa consommation par la cellule cérébrale. Nous avons étudié les effets de l'anoxie réalisée par une spoliation sanguine (Fig. 1) et par une hypotension provoquée par ganglioplégique (Fig. 2) sur le chat éveillé. Il résulte de ces 


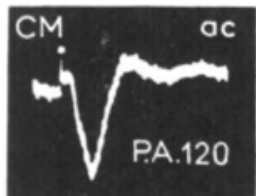

\section{Perfusion de Mêthioplégium}

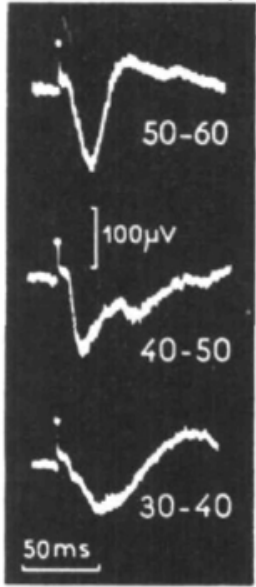

Figure 2. Chat éveillé. Centre Médian: Modification de l'activité évoquée après hypotension par ganglioplégique (methioplegium). Jusquà des chiffres de TA de $60 \mathrm{~mm}$. Hg, la réponse est maintenue. La durée de la réponse augmente ensuite; l'amplitude diminue lorsqu'on atteint des chiffres très bas.

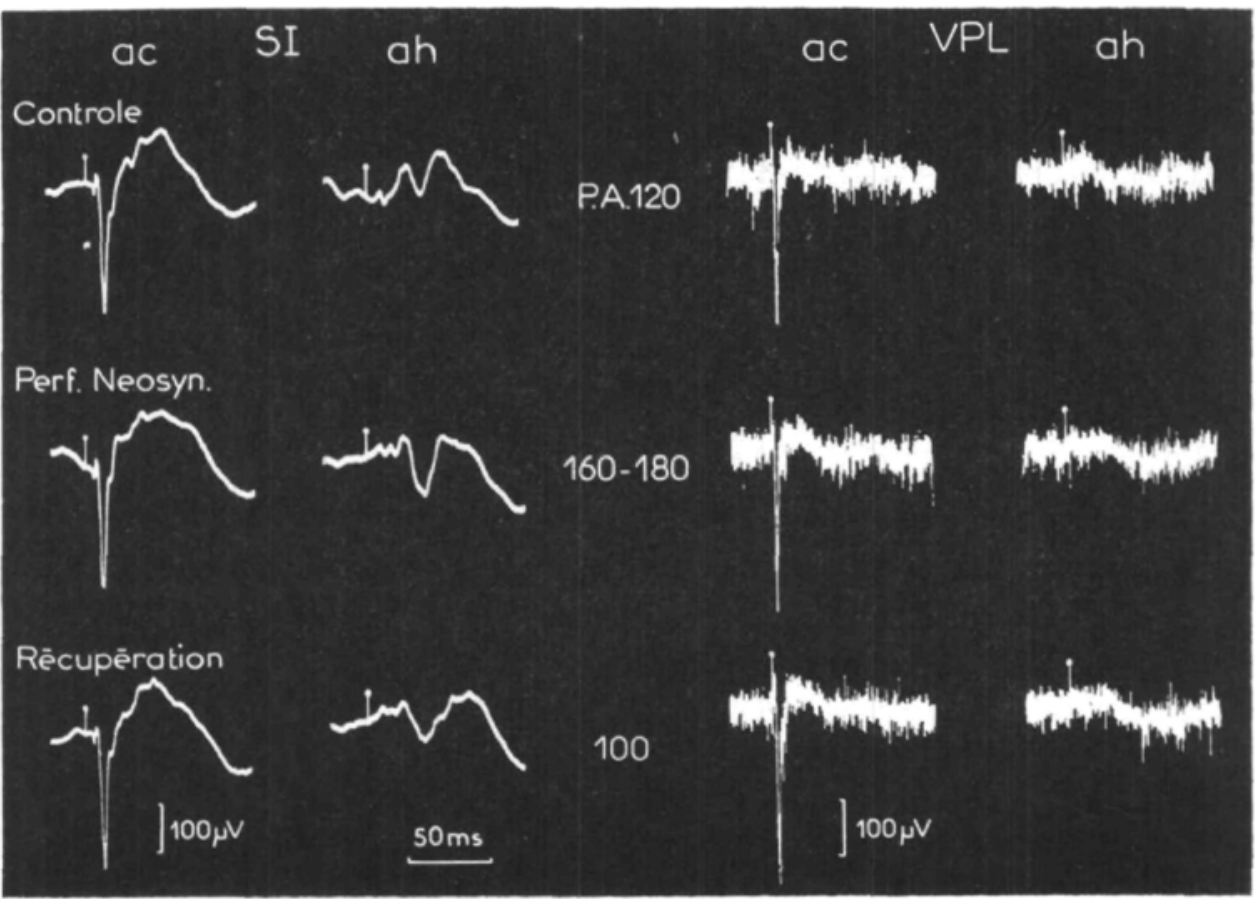

Figure 3. Chat éveillé: Hypertension réalisée par néosynéphrine. A gauche; activité évoquée au niveau de SI, par stimulation des membres antérieurs contro $(a c)$ et homolatéraux ( $a h$ ). A droite, activité évoquée au niveau du VPL, dans les mêmes conditions. 
VOURC'H et al.: L'ACTIVITÉ ÉLECTRIQUE CÉRÉBRALE

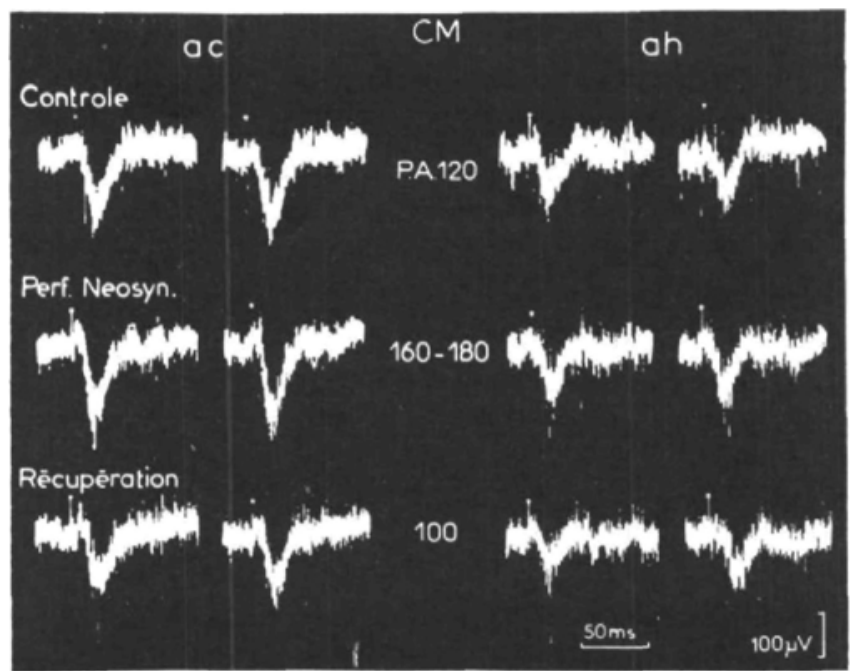

Figure 4. Mêmes conditions: Enregistrement dans le CM.

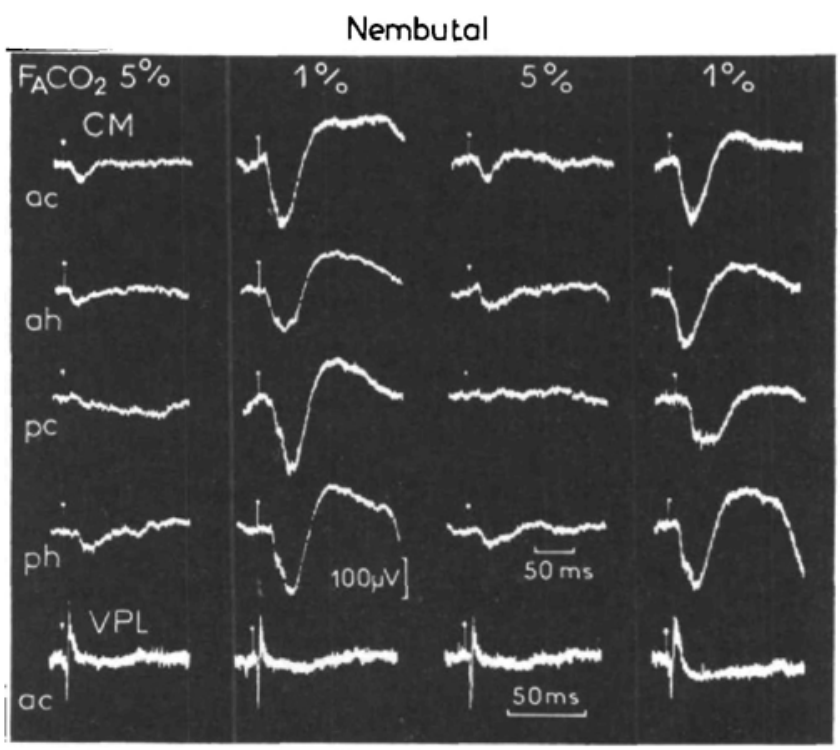

\section{Chlorolose}

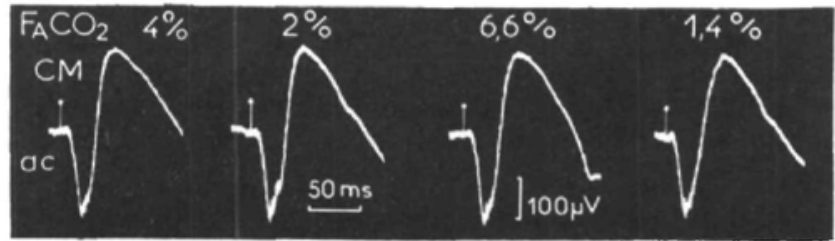

Figure 5. Modification des activités évoquées dans le thalamus, provoquées par des variations ventilatoires sur des chats anesthésiés au nembutal et au chloralose. Sous nembutal: l'activité évoquée enregistrée dans le $\mathrm{CM}$ s'accroit considérablement sous hyperventilation, et se réduit lors du retour à la normale. Le VPL ne varie pas. Sous chloralose: le tracé reste stable. 
études que l'activité électrique cérébrale, spontanée ou évoquée, est peu modifiée jusqu'à des chiffres tensionnels voisins de 60 ou $70 \mathrm{~mm}$. Hg. Au delà les réponses sont modifiées dans un ordre constant: le cortex est le plus sensible; puis les structures thalamiques associatives; enfin les centres thalamiques primaires. La restitution d'une pression artérielle normale, par perfusion de solutés macromoléculaires (polyvinyl pyrrolidone, Subtosan ${ }^{\circledR}$ ) permet une récupération dans les structures thalamiques primaires, mais les réponses restent déprimées dans les centres thalamiques associatifs et dans le cortex.

Par opposition, nous avons cherché à déterminer les effets de l'hypertension réalisée par les perfusions de néosynéphrine (Figs. 3 et 4 ), à crâne fermé. Nous n'y avons pas observé de modifications du tracé.

\section{Hyperventilation}

Outre l'alcalose respiratoire qu'elle provoque, l'hyperventilation réduit le débit sanguin cérébral et le volume de l'encéphale; la pression du liquide céphalorachidien s'abaisse.

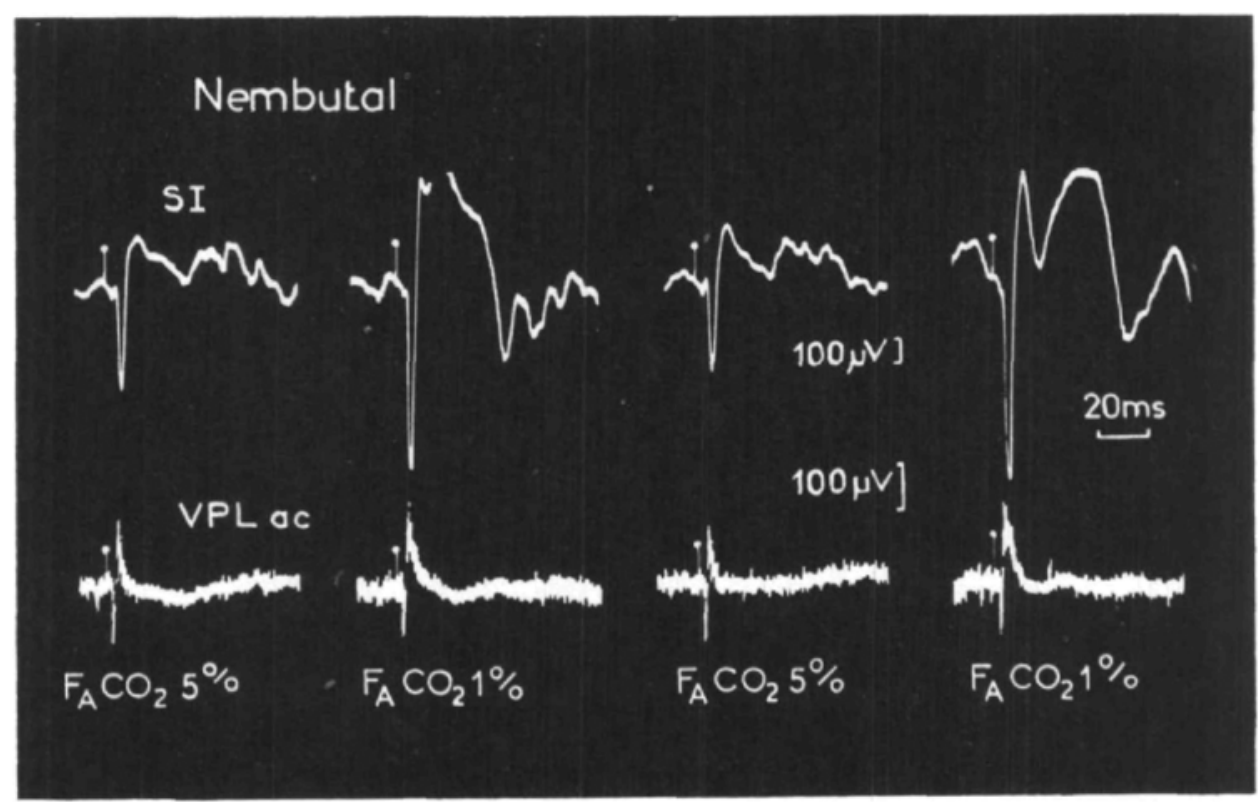

Figure 6. Chat trépané, sous nembutal. Même protocole expérimental. L'hyperventilation accroit considérablement la réponse dans SI. Celle du VPL n'est pas modifiée. (Le tracé est comparable à crâne fermé, mais l'amplitude est réduite.)

Les résultats varient selon le type d'agent anesthésique utilisé: sous pentobarbital, les structures thalamiques primaires sont peu modifiées, alors que les réponses associatives sont considérablement augmentées. Par contre, sous chloralose, le tracé n'est pas modifié (Fig. 5). 

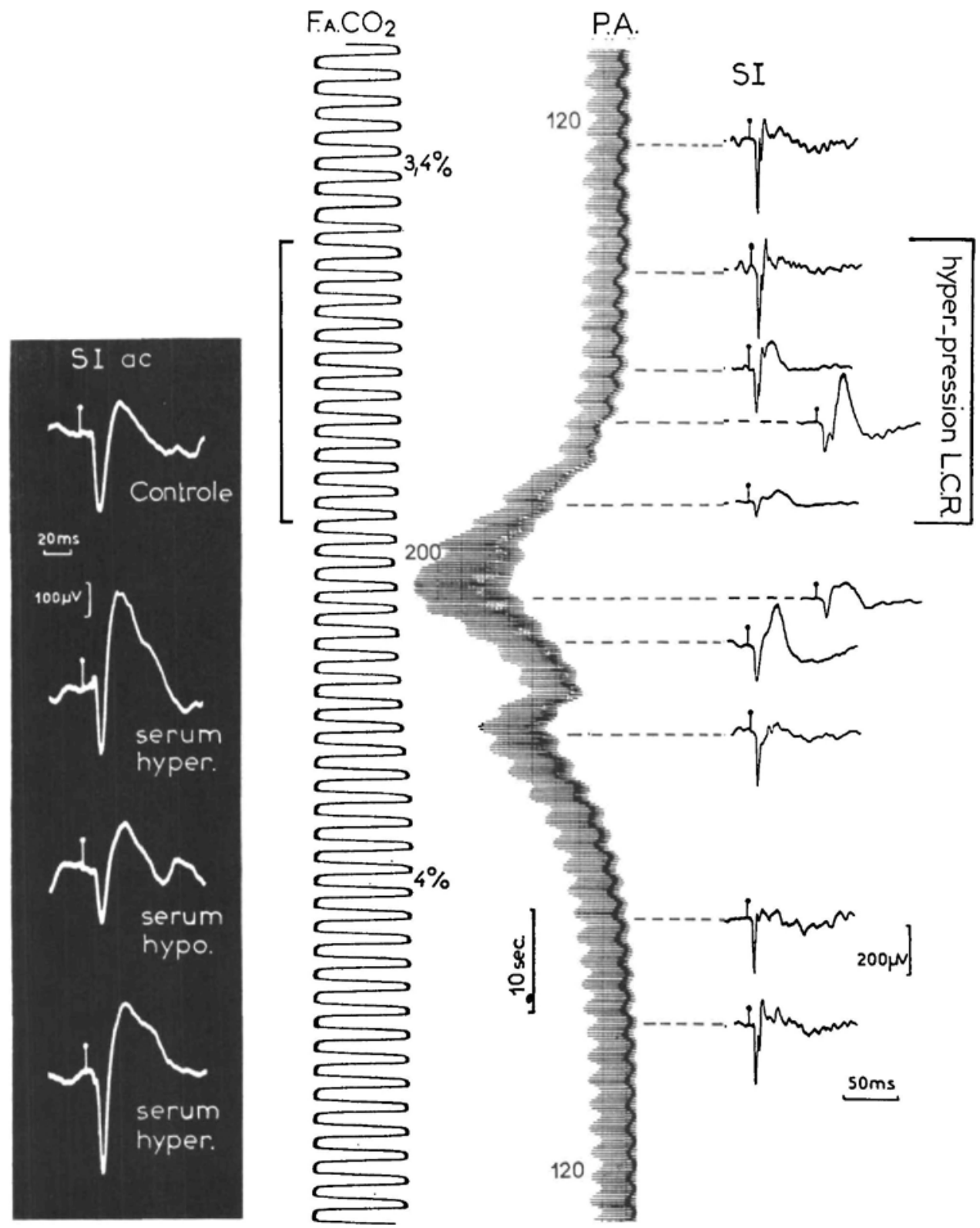

Figure 7 (à gauche). Chat trépané: Nembutal. Effet de l'injection intraveineuse de soluté hypertonique (CINA 20\%). Augmentation de l'activité évoquée, correspondant à un affaissement du cerveau. L'injection de soluté hypotonique (CINa 5\%o) rétablit la réponse à son niveau initial.

Figure 8 (à droite). Chat éveillé, crâne fermé. De gauche à droite: $\mathrm{F}_{\mathrm{A}_{\mathrm{CO}}}-\mathrm{PA}-\mathrm{SI}$. Modification des activités évoquées lors d’une hypertension intracranienne par injection de soluté de Ringer dans la grande citerne. L'amplitude des potentiels diminue bien avant l'hypertension artérielle qui en résulte. 
Au niveau du cortex, l'hyperventilation augmente considérablement l'amplitude des réponses somatiques (Fig. 6).

\section{Pression intracranienne}

La pression intracranienne peut modifier sensiblement le débit sanguin cérébral; la craniotomie, à elle seule, est susceptible de provoquer des différences du

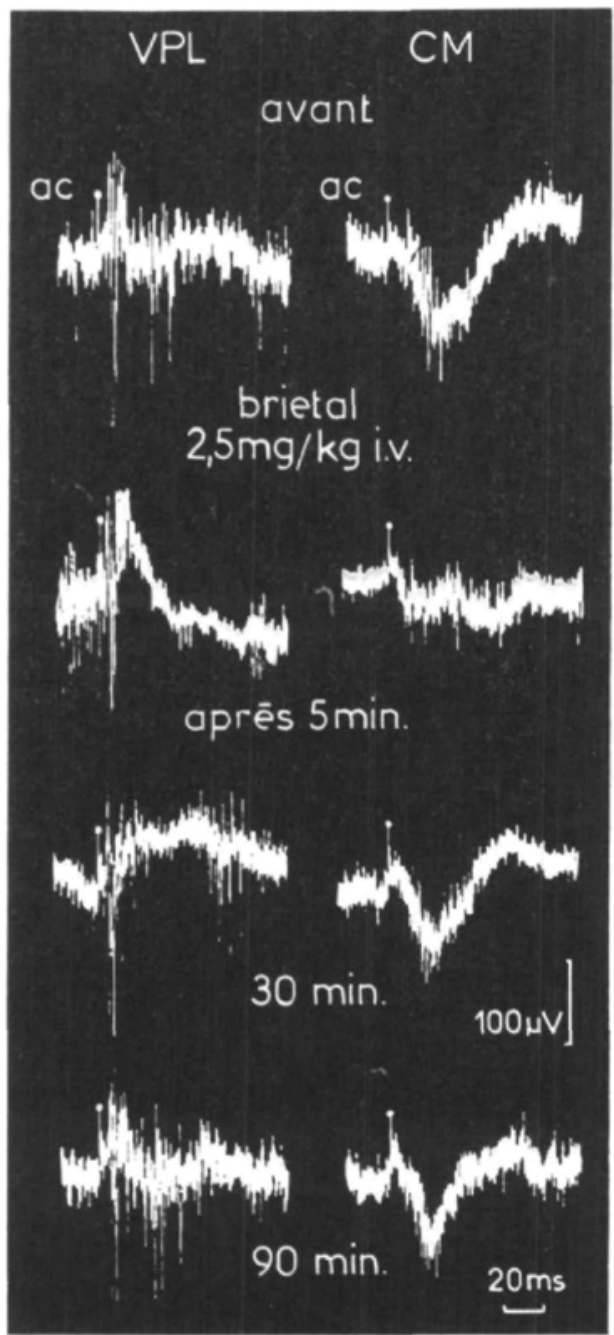

Figure 9. Chat éveillé en haut, sous Briétal ensuite. A gauche: VPL; à droite: CM. Effets de l'injection d'une dose de $2,5 \mathrm{mg}$. $\mathrm{kg}$, et récupération.

régime circulatoire, l'ablation du volet osseux agissant comme l'ouverture d'une soupape, modifiant les pressions intracraniennes.

L'injection de solutions hypertoniques entraîne une hypotonie cérébrale, et une 
augmentation des activités évoquées. L'injection de solutés hypotoniques rétablit l'état antérieur (Fig. 7).

Il en va de même si l'hypertension est réalisée par l'injection de soluté isotonique dans les citernes de la base (Fig. 8), l'hypertension intracranienne ainsi réalisée provoque une diminution des potentiels évoqués, antérieure à la réponse circulatoire (hypertensive) qui en résulte.

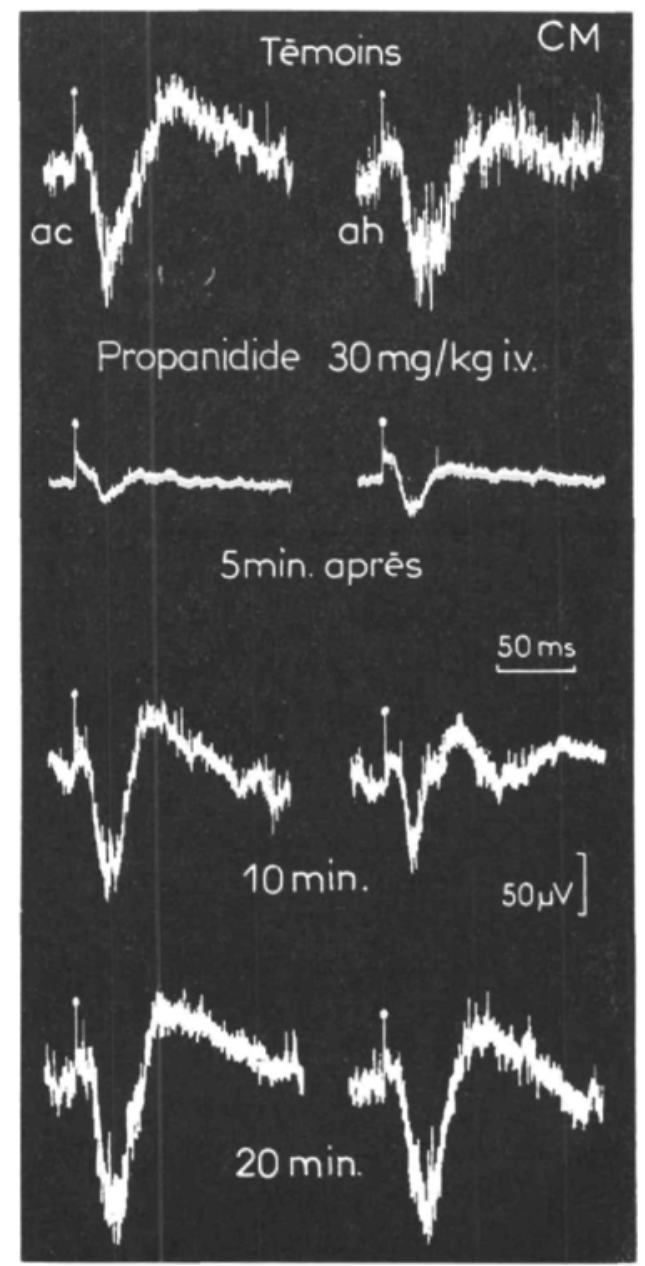

Figune 10. Même protocole. Effet de l'injection de propanidide sur le CM. Noter la brève durée d'action.

\section{ETUDE DE DROGUES NOUVELLES}

1. Nous avons cherché à étudier les effets de deux agents anesthésiques intraveineux à action courte de formule très différente; l'un, le méthohexital (Briétal ${ }^{\circledR}$ ), 
barbiturique; l'autre, le propanidide (Epontol ${ }^{\circledast}$ ) dérivé de l'eugenol. En clinique, la durée d'action de ces deux corps est sensiblement la même (5 à 10 minutes). Il peut être utile, pour le neurophysiologiste, de disposer de drogues d'actions brèves, qui permettent d'effectuer certaines investigations, sans compromettre de manière durable les réponses de la préparation. Il en va de même en pratique anesthésique.

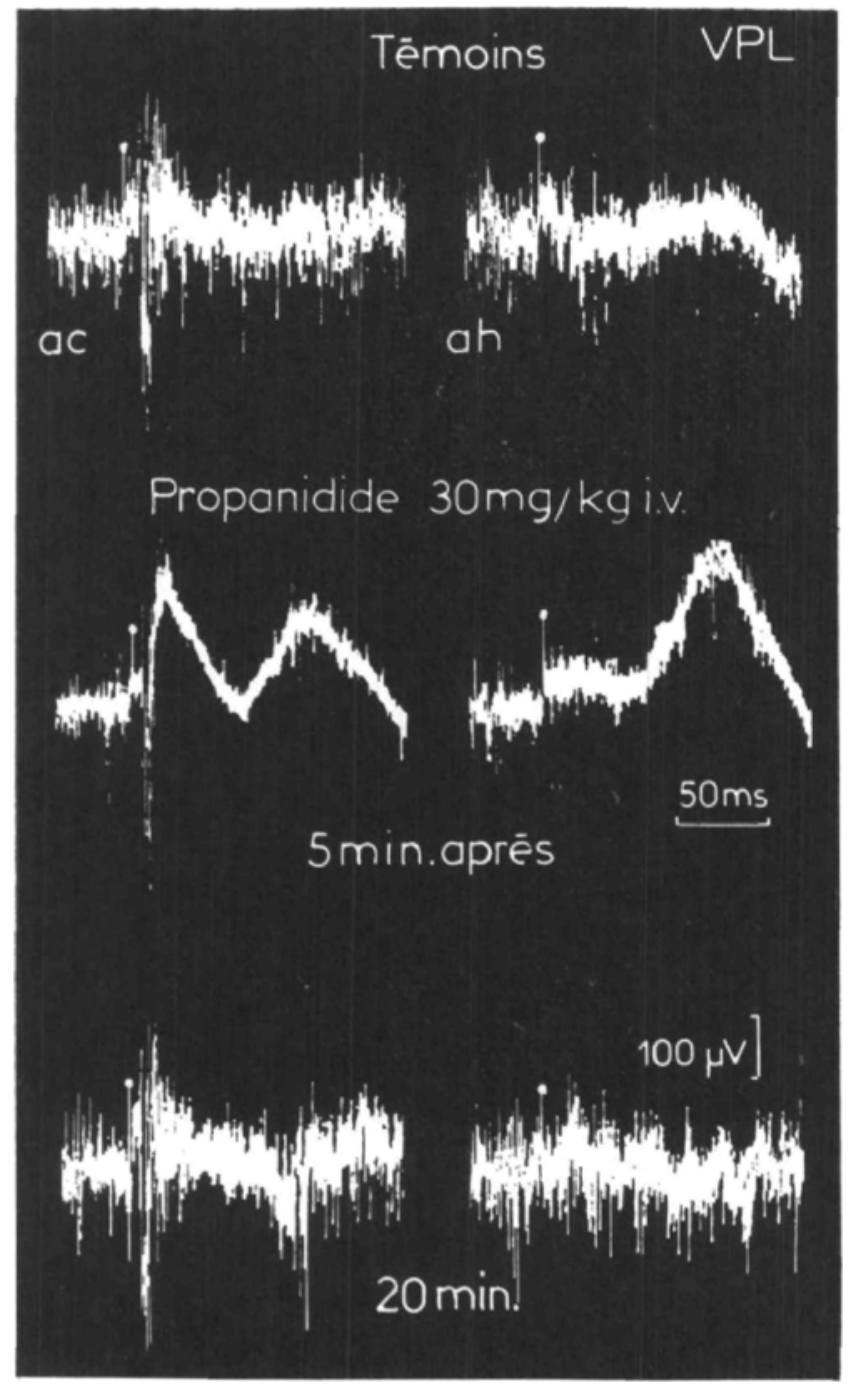

Figure 11. Même protocole. Enregistrement dans le VPL. A gauche: Stimulation du membre antérieur controlateral. A droite: Stimulation du membre antérieur homolateral. Pas de modification dé la réponse à brève latence; mais il apparaît une onde négative de plus longue latence lors de la stimulation des deux membres. 
Le méthohexital ne modifie pas les réponses à courte latence des structures thalamiques primaires, mais fait apparaître une onde négative de latence plus longue, en même temps que décroit l'activité spontanée. La réponse des structures associatives est très réduite (Fig. 9). La récupération est relativement rapide.

Le propanidide donne des résultats semblables (Figs. 10 et 11 ); on doit observer que ces agents, comme d'autres d'ailleurs, font apparaitre des fuseaux, ce qui peut amener des erreurs d'interprétation: la stimulation d'un membre homolatéral peut s'inscrire sur le tracé de telle manière qu'elle paraisse correspondre à une réponse dans une structure primaire. Toutefois, nous avons observé ce phénomène avec une fréquence qui nous semble trop grande pour être le fait d'une coïncidence.

\section{La Morphine et les morphinomimétiques}

La morphine sert évidemment de référence à toute étude portant sur les analgésiques centraux. Mais récemment, des produits nouveaux, plus puissants et plus maniables, parce que d'action plus brève, ont été synthétisés: la péthidine, la phénopéridine, le phentanyl. ${ }^{17}$

Ces trois drogues ont été étudiées sur le chat, curarisé et ventilé, et leur action est très voisine. Ils entrainent une augmentation des réponses évoquées, thalamiques et corticales, tant primaires qu'associatives. Cette augmentation de la réponse est abolie par la nalorphine.

Nous avons eu la surprise de constater que les effets de la morphine sur le chat, sont tout différents: les réponses associatives sont diminuées, et retrouvent leur amplitude initiale après nalorphine. Nous n'avons pas d'explication précise à donner pour rendre compte de cette singulière différence; toutefois, il faut remarquer que la morphine excite le chat, au lieu de le déprimer; cela peut éventuellement réduire les réponses associatives, dont on sait qu'elles dépendent de l'état de vigilence du sujet.

\section{Les Neuroleptiques}

Il en existe un grand nombre. Notre étude a été réalisée sur l'homme (en général des sujets parkinsoniens soumis à un contrôle électroencéphalographique pré-opératoire), et a porté sur le dehydrobenzpéridol (Dropéridol ${ }^{\circledR}$ ) et un produit voisin, d'action plus courte, la Spiramide.

Outre l'E.E.G., on a enregistré l'électrocardiogramme, la respiration, le tremblement (Figs. 12, 13, 14).

Pour des doses moyennes ( 5 à $10 \mathrm{mg}$. en injection intraveineuse, pour le dehydrobenzpéridol, et pour la Spiramide), on constate, après quelques minutes, un arrêt du tremblement, un état d'indifférence, un retard de la réponse. Mais le sujet ne dort pas, et son attention peut-être stimulée par des questions, des bruits; cette stimulation provoque une réapparition du tremblement.

Le dehydrobenzpéridol, pour une posologie moyenne, a une durée d'action de 1 à 2 heures; la Spiramide, de 30 minutes à une heure. A doses plus fortes, on peut observer des effets secondaires indésirables: impression de tête lourde, 
manque de concentration, pendant 12 et même 24 heures. La posologie doit donc rester prudente.

\section{ETude Clinique}

En neurochirurgie stéréotaxique, et plus spécialement dans le traitement de la maladie de Parkinson, sous contrôle neurophysiologique, les exigences opératoires rendent le choix d'une technique anesthésique délicat. Le sujet doit être calme; son tremblement doit être contrôlé pour ne pas gêner l'opérateur, tout en étant reproduit lorsqu'on fixe l'attention du malade; la respiration, la circulation ne doivent pas être déprimées; les drogues ne doivent pas gêner la lecture du tracé encéphalographique.

L'électroencéphalogramme est enregistré, d'une part à l'aide d'électrodes implantées dans le scalp, pour receuillir le tracé de surface; d'autre part à l'aide d'une électrode bipolaire introduite par un trou de trépan (en général occipital) et dirigée vers les structures thalamiques que l'on veut explorer. Un haut parleur est branché en dérivation et permet de suivre la progression de l'électrode; les activités électriques recueillies par l'électrode de profondeur sont enregistrées simultanément sur bande magnétique et sur le tracé encéphalographique de surface.

Initialement, nous avons utilisé l'hydroxydione, dont les effets se rapprochent beaucoup de ceux du chloralose, employé par les neurophysiologistes. ${ }^{14-16}$ Bien qu'effectivement cet agent se soit révélé utile et ait permis un enregistrement convenable, nous l'avons abandonné à cause de son maniement difficile: latence assez longue entre l'injection de la drogue et son action: thromboses veineuses; et aussi apparition, dans certains cas, de secousses musculaires rappelant celles observées sous chloralose.

Récemment, une technique anesthésique nouvelle a été mise au point associant un analgésique puissant (phenopéridine ou phentanyl) à un neuroleptique (dehydrobenzperidol ou Spiramide), sous le nom de neuroleptanalgésie. Cette association procure au malade une sensation d'indifférence, de calme, sans entrainer de sommeil véritable, au prix d'une dépression respiratoire modérée. Des prélèvements de sang artériel effectués sur cinq malades en cours d'opération nous ont montré que les modifications humorales (saturation en oxyhémoglobine, $\mathrm{pH}, \mathrm{PCO}_{2}$ étaient faibles ou absentes; si la dépression respiratoire devient excessive, la nalorphine en est un antidote parfait.

Les études expérimentales et cliniques ${ }^{17-19}$ nous ont montré que cette association de drogues était compatible tant avec des conditions opératoires satisfaisantes, pour le chirurgien et le malade, qu'avec un enregistrement électrique convenable; le tracé électroencéphalographique est peu modifié, et reste interprétable; les activités évoquées sont conservées (Figs. 15-20). L'identification des structures cérébrales rencontrées par l'électrode a toujours été facile; en particulier les représentations somatotopiques des différentes régions du corps, dans le thalamus, ont pu être précisées, grâce aux potentiels évoqués, ainsi que les relations entre le tremblement et les rythmes thalamiques. 


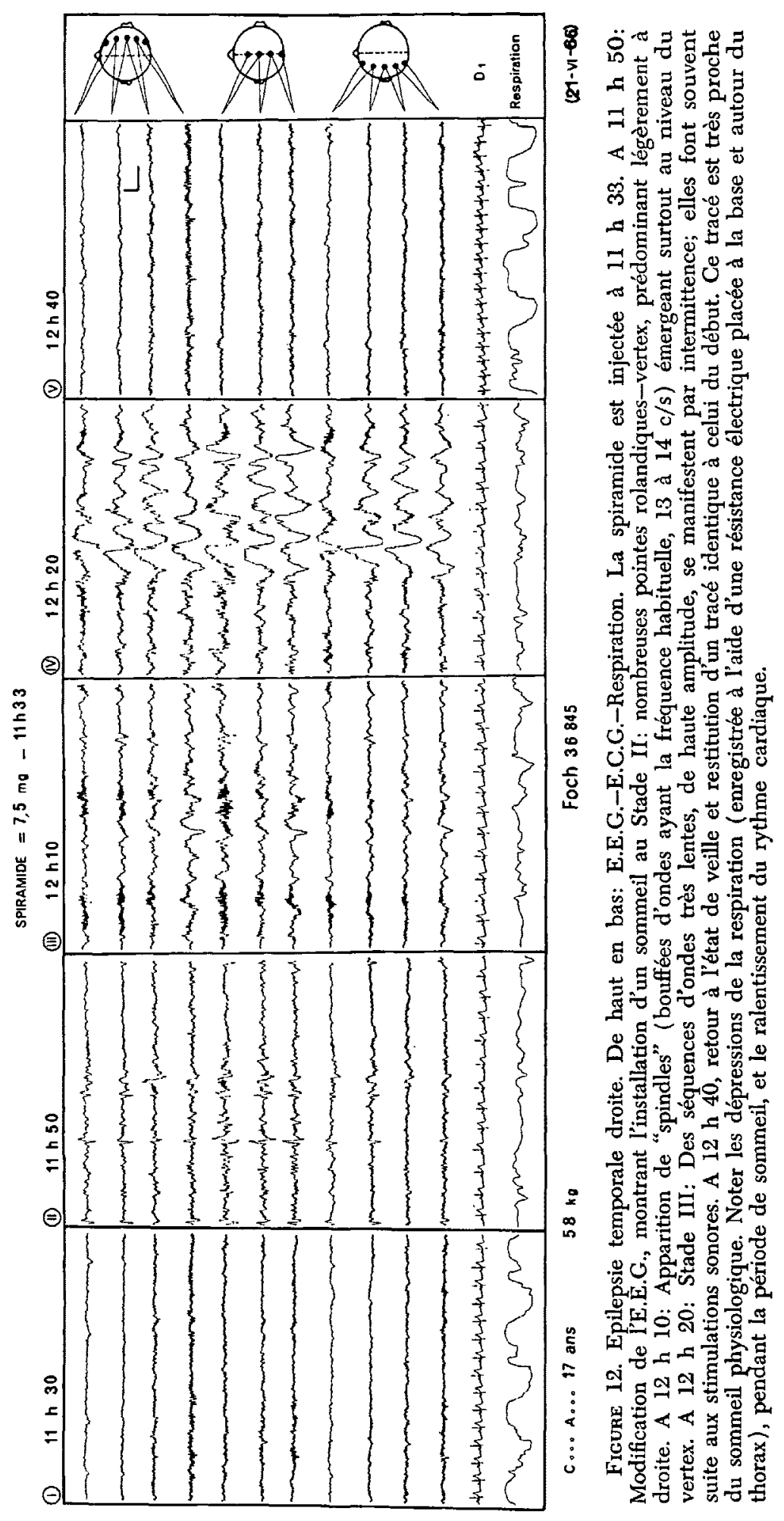




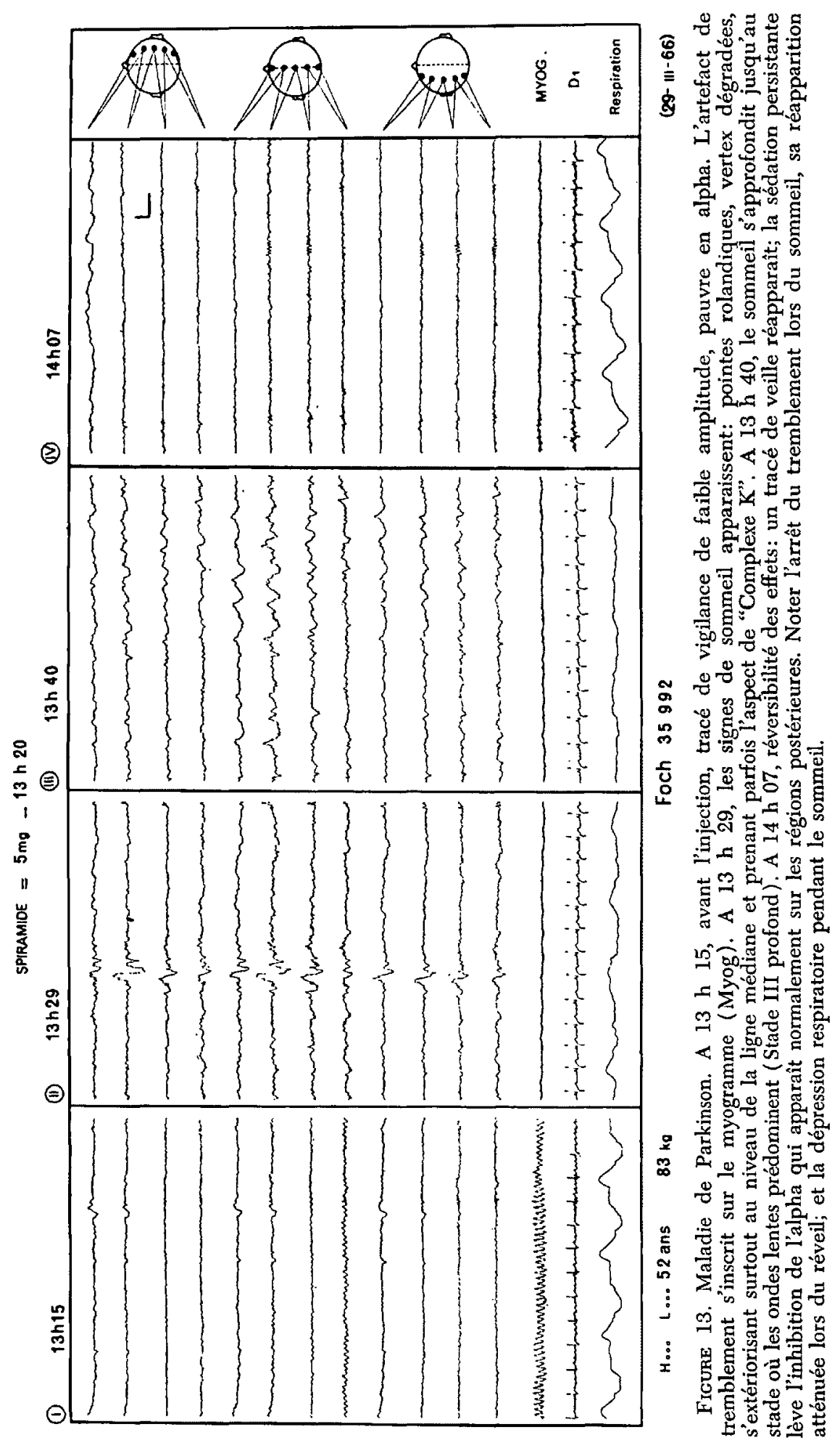




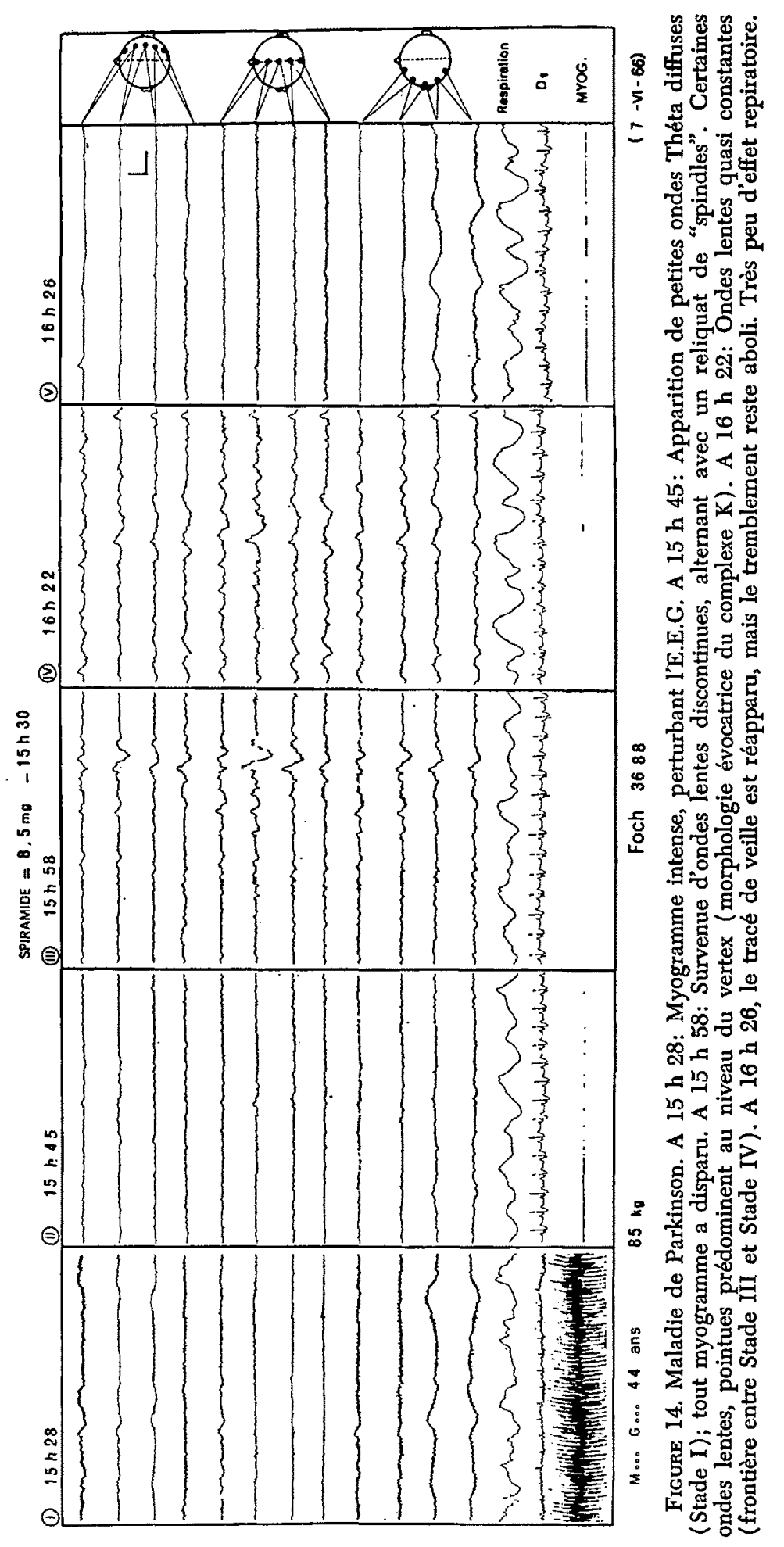




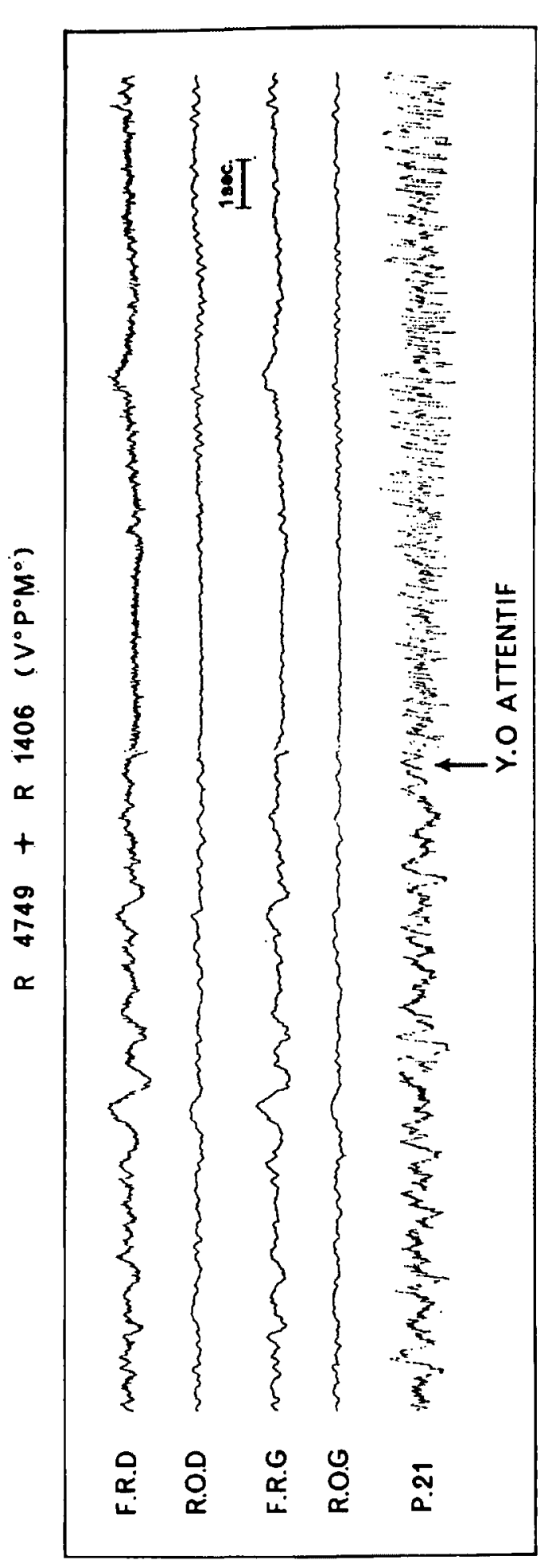

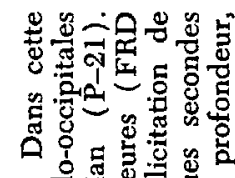

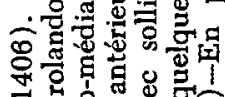

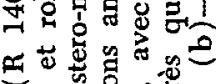

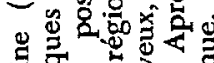

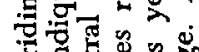
势 苟实 =

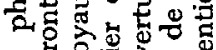

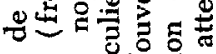

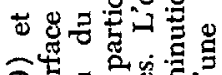

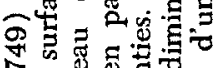

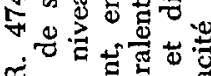

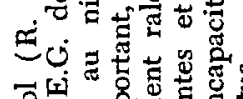

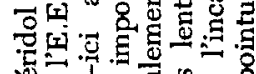

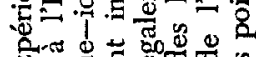

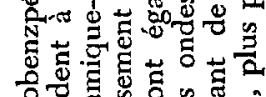

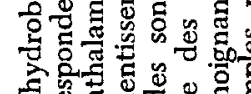

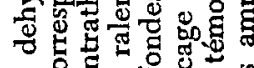
영

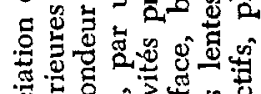

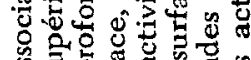

要

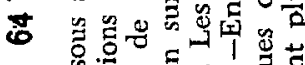

$\infty 5$

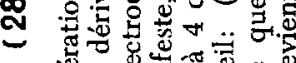

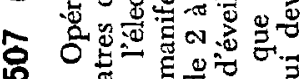

品

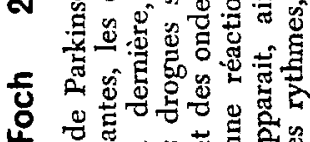

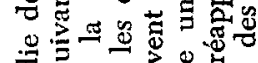

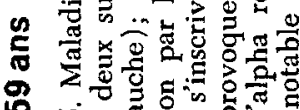
20

4

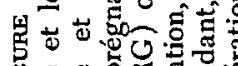

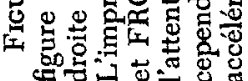




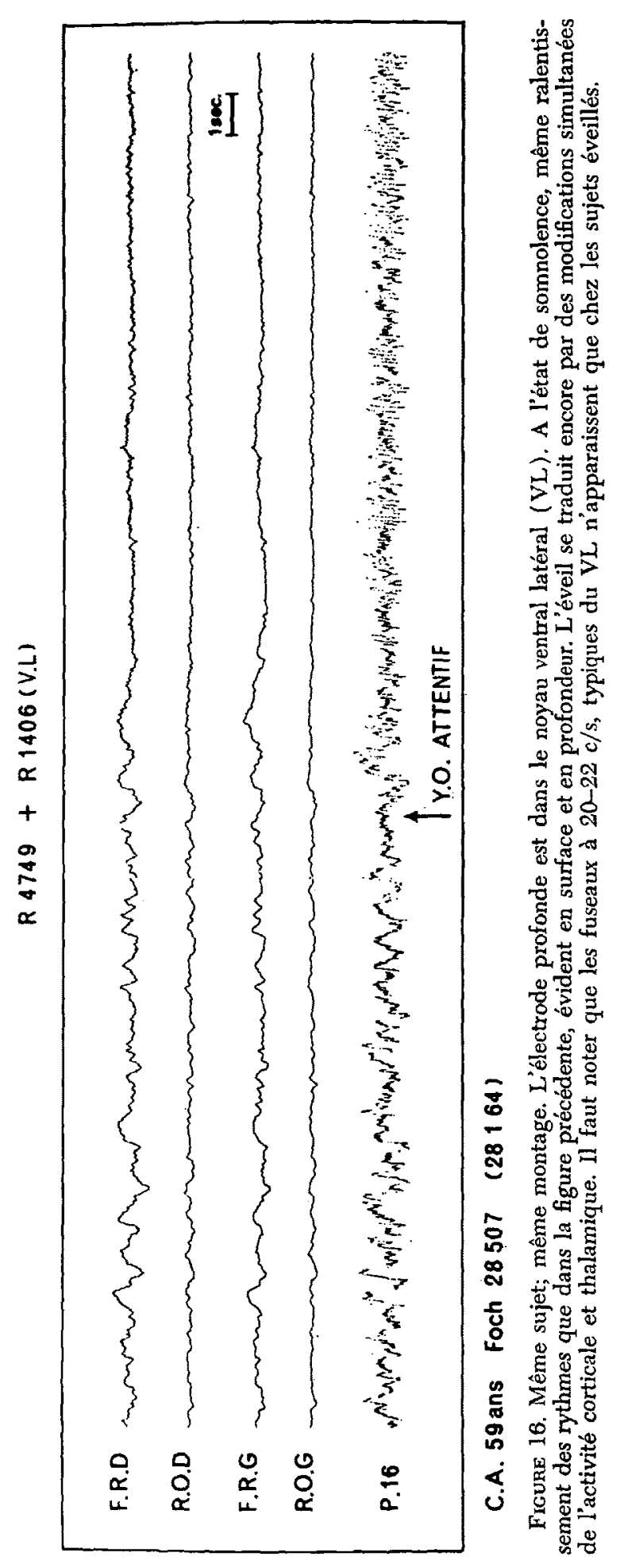




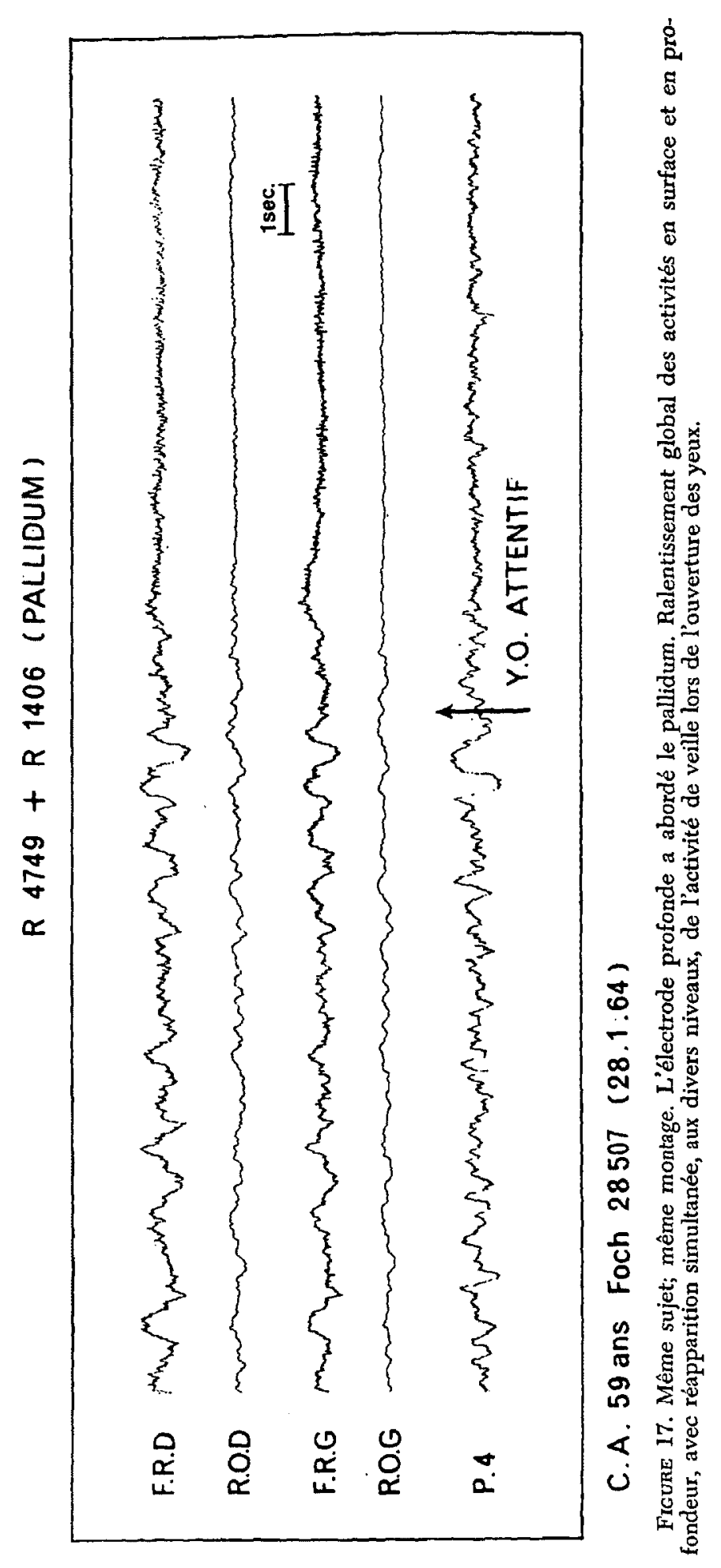




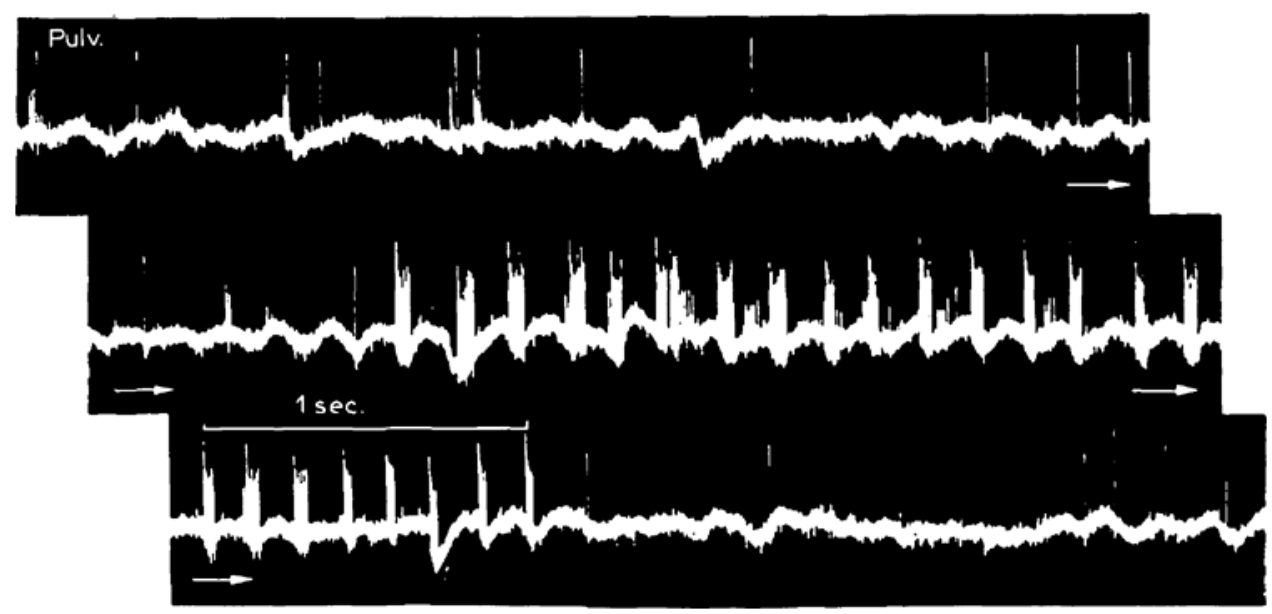

FIGURe 18. Même conditions opératoires. Enregistrement dans le pulvinar, montrant deux unités, presque silencieuses entre les "spindles", s'activant avec les ondes lentes des spindles.

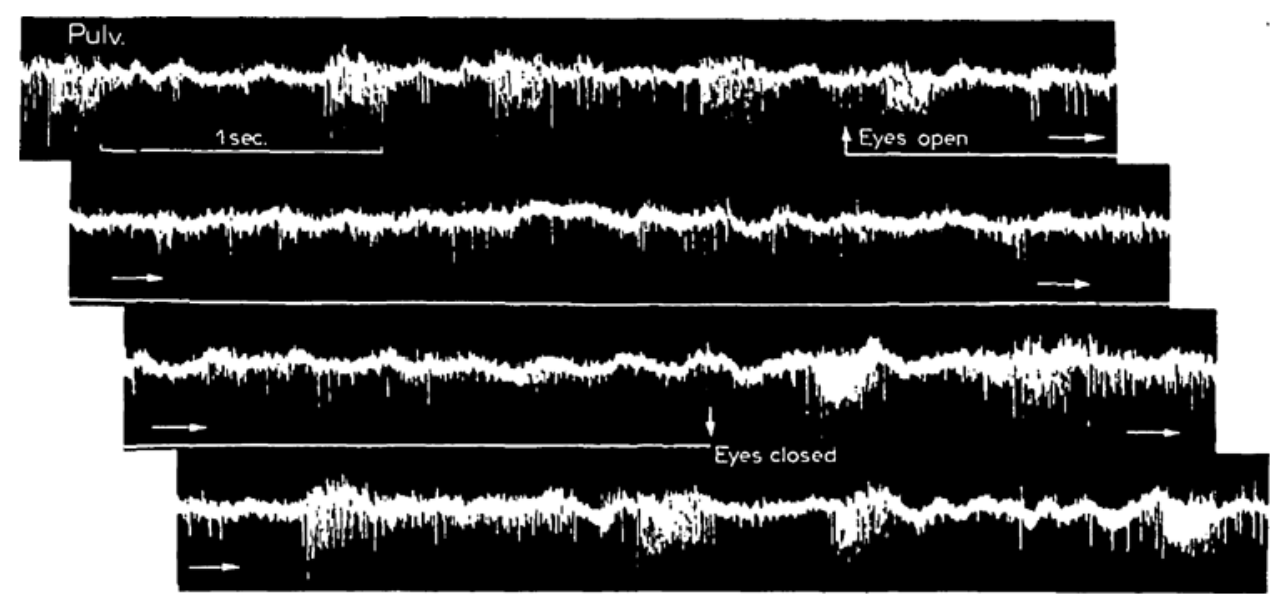

Figure 19. Mêmes condition opératoires. Décharges d'une activité, dans une unité du pulvinar, qui disparait quand le malade ouvre les yeux sur ordre.

\section{Cas particuliers}

\section{Traitement chirurgical de l'épilepsie}

Qu'il s'agisse de l'excision d'une zone corticale épileptogène, ou de coagulation amygdalienne, la durée de l'opération, et parfois l'état psychique du malade, imposent une anesthésie générale. Il est possible d'obtenir un enregistrement correct en utilisant une technique anesthésique appropriée.

Nous assurons l'induction de l'anesthésie par une dose faible de barbiturique à action courte; le malade est intubé, curarisé avec de la gallamine et ventilé à 


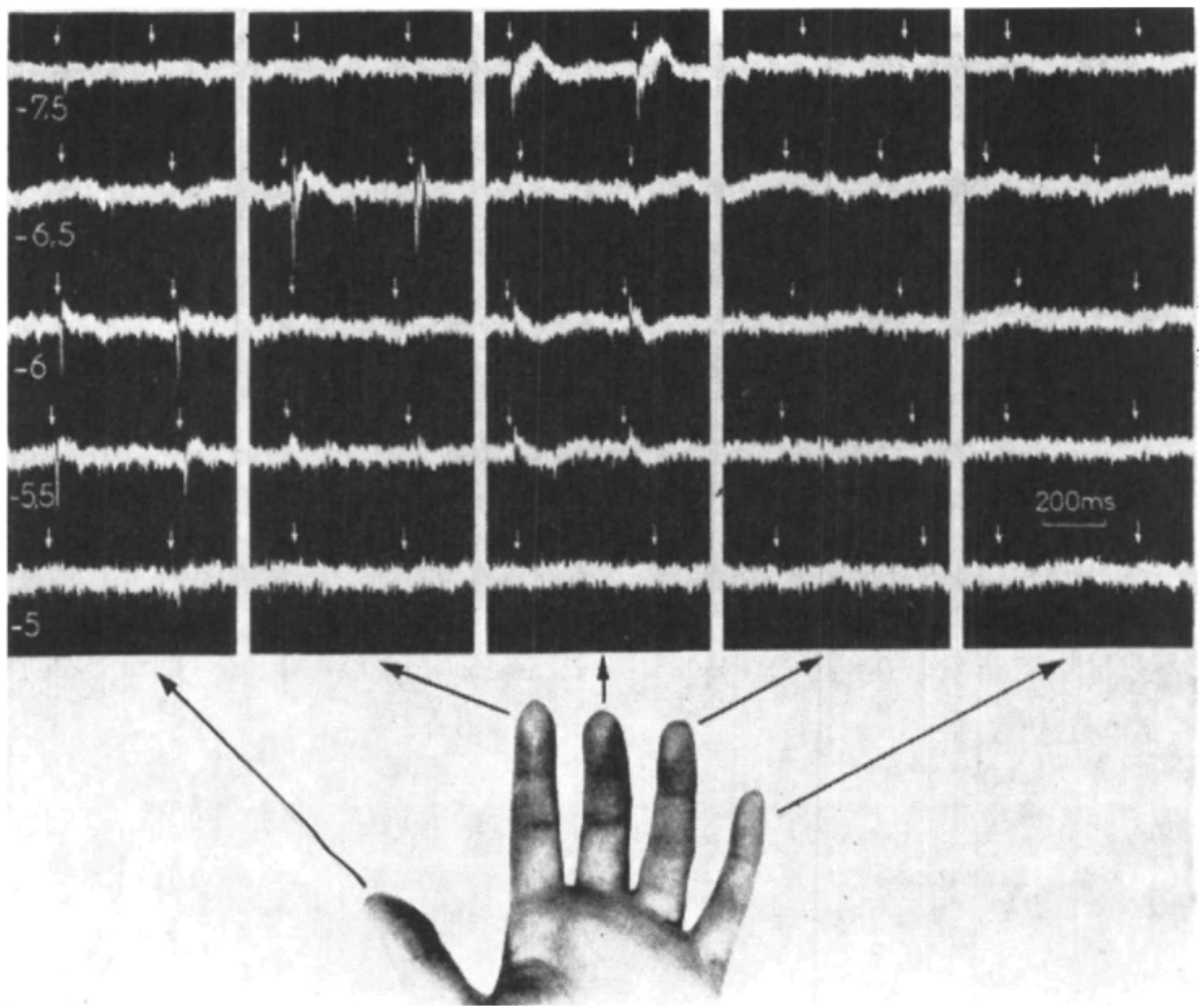

Fic. 20. Activités évoquées dans le noyau Ventral Postérieur à cinq niveaux différents, espacés de 0,5 à 1 millimètre. Stimulation tactile répétitive des doigts de la main controlatérale. En P-7,5, seule est obtenue la réponse pour le 3ème doigt. En P-6,5, la stimulation de l'index est la plus efficace. En $\mathrm{P}-6$, et en $\mathrm{P}-5,5$, pouce. En $\mathrm{P}-5$, réponses atténuées.

l'aide d'un mélange de protoxyde d'azote et d'oxygène. Au moment de l'enregistrement, on injecte de la phénopéridine et du dehydrobenzperidol, et on arrête l'administration de protoxyde d'azote. Ce gaz s'élimine rapidement (en 10 minutes à peu près); le tracé devient alors proche de l'état de veille (Figs. 21-23, pp. 540-41).

On peut également administrer de l'halothane au lieu de protoxyde d'azote; son élimination est également rapide.

\section{Topectomie}

Chez des malades souffrant d'algies incurables, on a cherché à repèrer, sur le cortex; la zone de projection correspondant à la partie du corps où siègent les douleurs, pour réaliser des éxèrèses limitées. Le cortex étant plus sensible aux agents anesthésiques que le thalamus, il importe de choisir un agent qui ne déprime pas les activités évoquées. Les malades sont endormis comme dans le cas précédent, à l'aide d'un barbiturique, intubés et ventilés: l'hydroxydione, la phénopéridine ont été utilisés lors des enregistrements. Il faut avouer toutefois, que les résultats ont été décevants, tant sur le plan de la qualité des potentiels receuillis, que sur celui du résultat opératoire. 


\section{RÉSUMÉ ET CONCLUSION}

Dans le cadre d'une étude systématique de l'action des anesthésiques généraux sur les structures cérébrales, nous avons étudié, d'une part les perturbations provoquées par des modifications respiratoires et circulatoires, ainsi que l'action d'agents anesthésiques intraveineux à action brève-morphine et morphinomimétiques-sur l'animal; d'autre part, l'action de certaines neuroleptiques chez l'homme.

Les données ainsi receuillies ont été appliquées en clinique et dans le traitement chirurgical de la maladie de Parkinson, de certaines formes d'épilepsie, et de topectomies pour algies rebelles, sous contrôle neurophysiologique. Un choix judicieux des drogues anesthésiques permet d'obtenir des conditions opératoires satisfaisantes, tout en étant compatible avec un enregistrement correct des activités spontanées ou évoquées dans les structures cérébrales explorées.

\section{SUMMARY}

Within a systematic investigation of the action of the general anaesthetic agents on cerebral structures, we have studied, on one hand, the modifications produced by circulatory and respiratory changes and also the action of anaesthetic agents: i.v. short acting, morphine, and morphino-mimetics on the animal; on the other hand, we have studied the action of some neuroleptics on man.

The results obtained have been applied clinically in the surgical treatment of Parkinson's disease, in certain kinds of epilepsy, and in topectomies for intractable pain under neurophysiological control. An appropriate choice of anaesthetic drugs allows one to obtain satisfactory operative conditions and at the same time permits a correct record of the evoked and spontaneous activities in explored cerebral structures.

Réferences, voir p. 542 . 


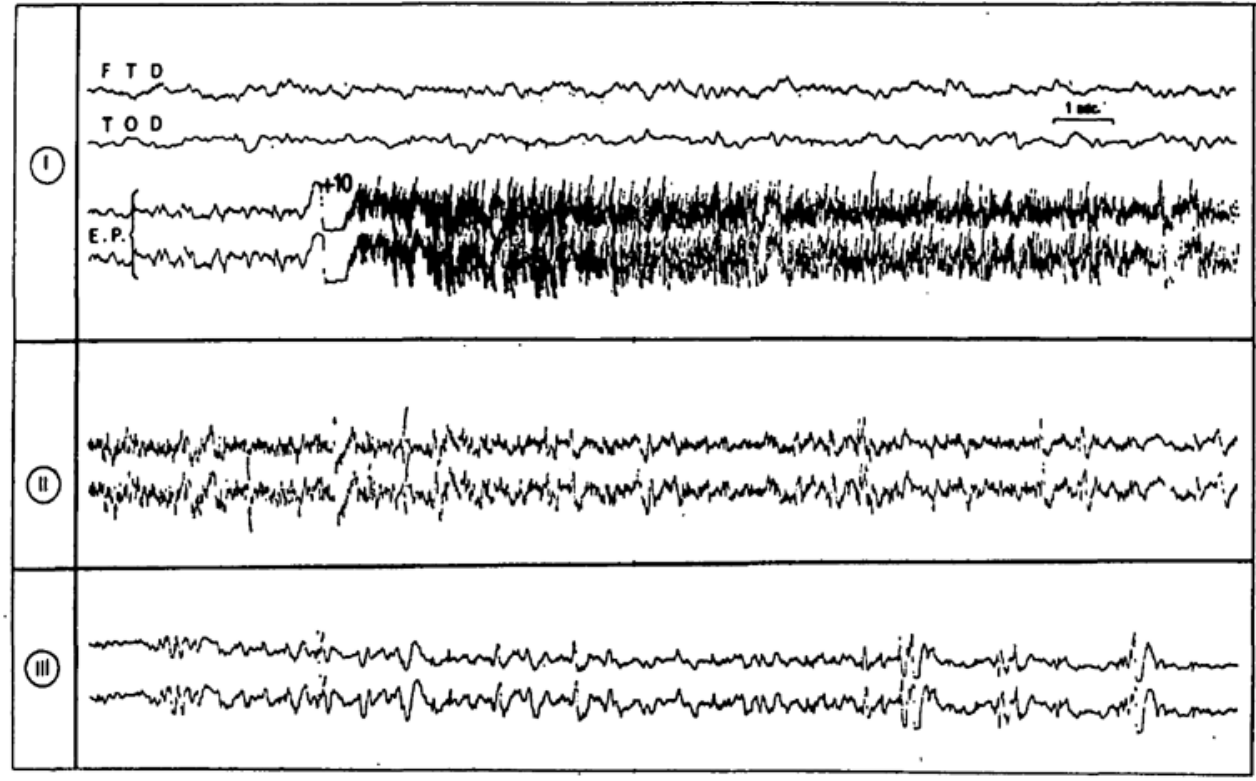
F... J.M.
13 ans
Foch 32262
15. III. 65

(I)




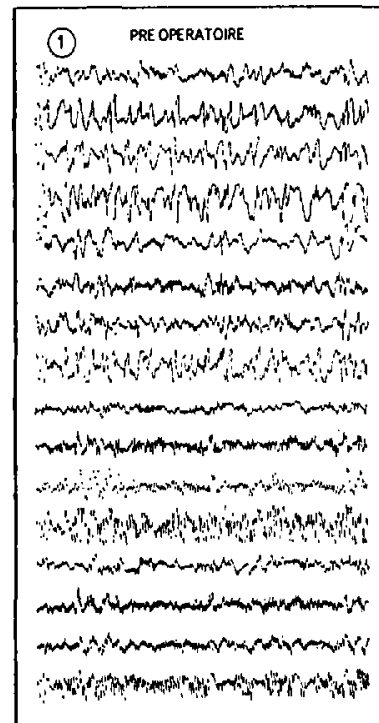

F... J.M. 13 ans

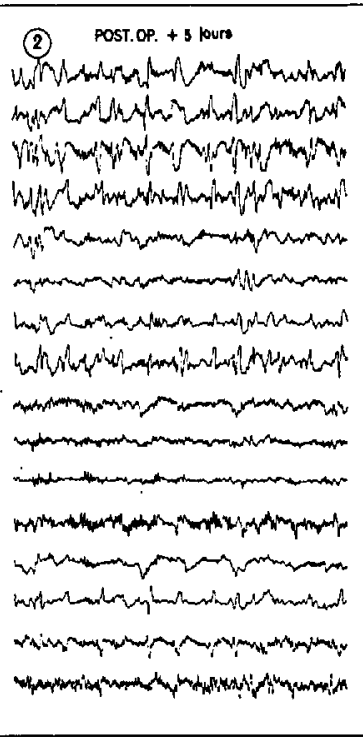

Foch $\quad 32132-327-354$

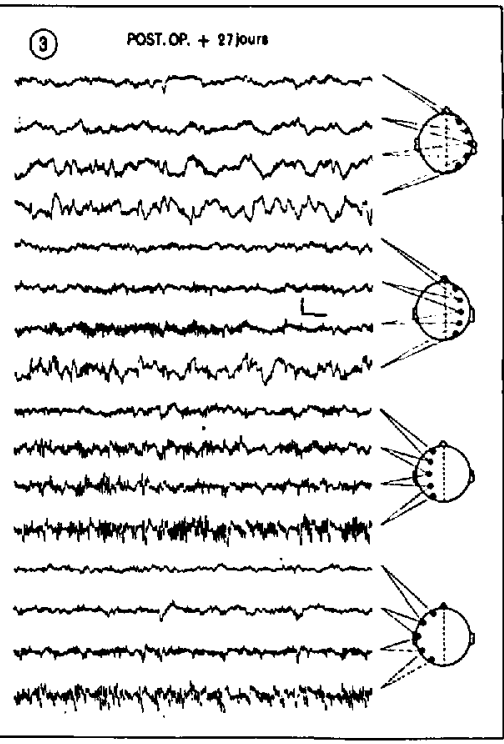

Foch mars_avril 65

Epilepsie temporale droite, évoluant depuis deux ans, avec instabilité psycho-motrice et troubles de l'attention. Traitement chirurgical par coagulation de l'amygdale. Les trois planches (Figs. 21, 22, p. 540; Fig. 23, p. 541) concernent le même malade.

Figure 21. Enregistrement simultané de l'E.E.G. de surface (les deux dérivations supérieures) et de l'activité profonde, au niveau amygdalien (sur les deux dérivations inférieures). En 1. Au moment de la progression de l'électrode profonde (EP), à +10 , brusque décharge amygdalienne du type habituel: activités de haute amplitude, très rapides (au-delà de $60 \mathrm{c} / \mathrm{s}$, pendant les premières secondes, puis ralentissement progressif). La décharge d'enfoncement devient beaucoup moins intense après une dizaine de secondes. En 2 et 3 . Seules ont été retenues, pour chaque fragment, les dérivatives amygdaliennes; en 2 , on voit s'achever une décharge d'enfoncement et se rétablir l'activité spontanée. Celle-ci est très anormale et comporte de décharges de pointes et pointe-ondes.

Figure 22. Pour les fragments 1 et 2, enregistrement simultané de l'E.E.G. de surface (les 4 dérivations supérieures) et de l'activité profonde (les 2 dérivations inférieures). L'électrode, cette fois, est poussée dans l'hippocampe $(+15)$ et la réaction d'enfoncement y est extrèmement vive comme on le voit en 1 . Aucune répercussion de la décharge profonde sur le tracé transcranien homolateral (FTD et TOD). En 2. Une minute après l'enfoncement de l'électrode, la réaction d'enfoncement s'est épuisée, démasquant le tracé spontané; celui-ci est constitué par de multiples décharges de pointe-ondes et polypointe-ondes.

Figure 23. Aspect pré- et post-opératoire. En 1. Avant l'intervention, les activités physiologiques sont très détériorées à droite; d'innombrables pointes et pointes-ondes émergent au niveau du lobe temporal, ainsi que des régions postérieures adjacentes. En 2. Cinq jours après coagulation amygdalienne droite, discontinuité de l'activité paroxystique, toujours importante cependant; quelques pointes isolées apparaissent sur le lobe temporal gauche. En 3. Vingt sept jours après l'intervention, disparition des pointes; constitution à droite d'un bon alpha. II persiste toutefois des ondes très lentes, polymorphes, au niveau de la région temporale droite (surtout postérieure) et du lobe occipital voisin. 


\section{REFERENCES}

1. Mécanisme de la narcose. Colloques Internationaux du Centre national de la Recherche Scientifique-1951. C.N.R.S., 13, quai Anatole France, Paris VIIème.

2. KuPFer; Tsoucaris, D.; \& Tsoucaris, J. Contribution à l'étude moléculaire de la narcose. Anesth. Analg. Réanim. 23: 151 (1966).

3. Harris, T. A. B. The Mode of Action of Anaesthetics. E. \& S. Livingstone, ed., 1951.

4. Faulconner, A. \& Bickford, R. Electroencephalography in Anesthesiology. C. Thomas, ed., 1960 .

5. BRECHNen, V. \& Walter, R. Practical Electroencephalography for the Anesthesiologist. C. Thomas, ed., 1962.

6. Wyke, B. Principles of General Neurophysiology Relating to Anaesthesia and Surgery. Butterworth, ed., 1959.

7. Guiot, G.; Brion, S.; Rougerie, J.; Hertzog, E.; Escourolle, R.; \& Sachs, M. La Destruction stéréotaxique du pallidus interne. Ann. chir. 12: 1003 (1958).

8. Guiot, G.; Hardy, J.; \& Albe-Fessard, D. Délimitation précise des structures souscorticales et identification des noyaux thalamiques chez l'homme par l'électrophysiologie stéréotaxique. Neurochirurgia. 5: 1 (1962).

9. Albe-Fessard, D.; Arfel, G.; Guiot, G.; Hardy, J.; Vourc'h, G.; Hertzog, E.; Aleonard, P.; \& Derome, P. Dérivations d'activités spontanées et évoquées dans les structures cérébrales profondes de l'homme. Revue Neurol. 106: 89 (1962).

10. Albe-Fessard, D.; Arfel, G.; \& Guiot, G. Activités électriques caractéristiques de quelques structures cérébrales chez l'homme. Ann. chir. 17: 1185 (1963).

11. Albe-Fessard, D.; Arfel, G.; Gutot, G.; Derome, P.; Hertzog, E.; Vourc'h, G.; Brown, H.; Aleonard, P.; de la Herran, J.; \& Trigo, J. C. Electrophysiological Studies of Some Deep Cerebral Structures in Man. J. Neurol. Sc. 3: 37 (1966).

12. Denavit, M. Action différencielle de quelques anesthésiques sur les structures alimentées par les voies spécifiques et les voies associatives. Anesth. Analg. Réanim. 20: 747 (1963).

13. WYKE, B. Brain Function and Metabolic Disorders. Butterworth, ed., 1963.

14. Vourc'r, G.; Hardy, J.; \& Denavir, M. Problèmes anesthésiques posés par l'exploration thalamique et corticale en neurochirurgie. Acta Institut Anesth. 11: 115 (1962).

15. - - Problems Raised by Anaesthesia for Thalamic and Cortical Exploration in Neurosurgery. Brit. J. Anaesth. 35: 208 (1963).

16. Vourc' H, G. \& ARFel, G. Action de certains agents anesthésiques sur l'activité électrique cérébrale. Actualités Pharmacologiques. 17ème série (1964), p. 213.

17. Vourc'H, G.; Arfel, G.; Brown, H.; Millan, E.; \& Besson, J. M. Anesthesia for Stereotactic Neurosurgery. International Anesthesiology Clinics. European Trends in Anesthesiology. Little, Brown \& Co., 1965. Vol. 3, p. 705.

18. Albe-Fessard, D.; Arfex, G.; Gutot, G.; Derome, P.; \& Gulbaud, G. Thalamic Unit Activity in Man. Electroencephalog. \& Clin. Neurophysiol. Suppl. 25: 132 (1967). 